\title{
A prática da automedicação por universitários
}

\author{
The practice of self medication by university students \\ La práctica de la automedicación por parte de estudiantes universitários
}

Recebido: 26/06/2021 | Revisado: 04/07/2021 | Aceito: 05/07/2021 | Publicado: 16/07/2021

José Marcos da Silva Lima
ORCID: https://orcid.org/0000-0002-2057-4576
Universidade Federal de Campina Grande, Brasil
E-mail: marcosdyasbr@ gmail.com
Cláudio Gomes da Silva Júnior
ORCID: https://orcid.org/0000-0002-8384-9338
E-mail: cgsjunior.cso@gmail.com
Secretaria de Educação e Esporte do Estado de Pernambuco, Brasi
Sabrina Márcia Resende de Almeida Santos Cunha
ORCID: https://orcid.org/0000-0003-0135-3011
$4^{\text {a }}$ Gerência Estadual de Saúde, Brasil
E-mail: bina35cunha@gmail.com
Maria Isabel da Silva Lima
ORCID: https://orcid.org/0000-0003-2929-4527
Universidade Federal de Campina Grande, Brasil
E-mail: isabelldyas@ gmail.com
Elicarlos Marques Nunes
ORCID: https://orcid.org/0000-0003-2135-6017
Universidade Federal de Campina Grande, Brasil
E-mail: elicarlos.marques@ professor.ufcg.edu.br

\section{Resumo}

Esse trabalho objetiva evidenciar e discutir os dados descritos na literatura acerca da automedicação entre os universitários, bem como analisar os fatores que contribuem para essa prática. Nesse estudo foi realizada uma coleta no PubMed®, Google Acadêmico®, Literatura Latino-Americana em Ciências da Saúde e Science Direct, utilizando os descritores, automedicação e estudantes. Após o refino da busca e aplicação dos critérios de inclusão, foram compilados nessa revisão 23 artigos que fizeram referência ao tema desse estudo. Observou-se que 51,7\% dos estudantes de cursos da saúde fazem uso de medicamentos sem prescrição médica, mesmo possuindo total conhecimento dos riscos que podem trazer para saúde, a prevalência de automedicação entre os universitários variou de $36,1 \%$ a $99,5 \%$. Dentre as principais classes de medicamentos utilizadas, destacaram-se os anti-inflamatórios $23,3 \%$ e analgésicos $31,4 \%$. Os fatores que estão atrelados à prática de automedicação dos universitários são a acessibilidade para adquirir medicamentos sem prescrição médica, demora nos atendimentos do sistema de saúde pública, os valores elevados das consultas particulares e a grande disponibilidade de informações existentes na internet. Esse comportamento de se automedicar pode desencadear o surgimento de dores crônicas. Visto que ao utilizar um medicamento específico de maneira frequente, seu efeito deixa de ser percebido, fazendo com que o indivíduo passe a ingerir doses maiores. Diante disso, conclui-se que é necessário que seja implantando nas universidades métodos educativos, principalmente entre os acadêmicos dos cursos da saúde. Utilizando estratégias que conscientizem os mesmos e crie uma abordagem sobre o uso racional de medicamentos em disciplinas transversais ao longo da graduação.

Palavras-chave: Saúde pública; Estudantes; Fatores de riscos; Automedicação.

\begin{abstract}
This work aims to highlight and discuss the data described in the literature about self-medication among university students, as well as to analyze the factors that contribute to this practice. In this study, a data collection was carried out in PubMed ${ }^{\circledR}$, Google Acadêmico ${ }^{\circ}$, Latin American Literature in Health Sciences and Science Direct, based on the descriptors: self-medication and students. After refining the search and applying the inclusion criteria, 23 articles that referred to the theme of this study were compiled in this review. It was observed that $51.7 \%$ of students in health courses use drugs without a prescription, even though they have full knowledge of the risks that this practice can bring to health, the prevalence of self-medication among university students ranged from $36.1 \%$ to $99.5 \%$. Among the main classes of drugs used, anti-inflammatory drugs stood out $23,3 \%$ and analgesics $31,4 \%$. The factors that are linked to the self-medication practice among university students are the accessibility to purchase medications without medical prescription, the delay in the attendance of the public health system, the high values of private consultations and the great availability of information available on the internet. This self-medication behavior can trigger chronic pain incidences. Since the use of a specific medication on a frequent basis, can make its effect is no longer perceived,
\end{abstract}


causing the individual to ingest higher doses. Therefore, it is concluded that it is necessary to implement educational methods in universities, especially among academics in health courses. Using strategies that make them aware and create an approach on the rational use of medicines in transversal disciplines throughout the graduation.

Keywords: Public health; Students; Risk factor; Self medication.

\section{Resumen}

Este trabajo tiene como objetivo resaltar y discutir los datos descritos en la literatura sobre la automedicación en estudiantes universitarios, así como analizar los factores que contribuyen a esta práctica. En este estudio se realizó una recopilación en PubMed®, Google Acadêmico®, Literatura Latinoamericana en Ciencias de la Salud y Science Direct, con base en los descriptores, automedicación; estudiantes. Tras afinar la búsqueda y aplicar los criterios de inclusión, en esta revisión se recopilaron 23 artículos que hacían referencia a la temática de este estudio. Se observó que el $51,7 \%$ de los estudiantes de los cursos de salud consumen medicamentos sin prescripción médica, aunque tienen pleno conocimiento de los riesgos que pueden traer a la salud, la prevalencia de la automedicación entre los estudiantes universitarios osciló entre el 36,1\% y el 99,5\%. Entre las principales clases de fármacos utilizados destacan los antiinflamatorios el 23,3\% y los analgésicos el 31,4\%. Los factores que se vinculan a la práctica de la automedicación de los estudiantes universitarios son la accesibilidad para adquirir medicamentos sin prescripción médica, el retraso en la asistencia al sistema público de salud, los altos valores de las consultas privadas y la gran disponibilidad de información disponible. En Internet. Este comportamiento de automedicación puede desencadenar incidentes de dolor crónico. Ya que al usar un medicamento específico de forma frecuente, su efecto ya no se percibe, provocando que el individuo ingiera dosis más elevadas. Por tanto, se concluye que es necesario implementar métodos educativos en las universidades, especialmente entre los académicos en los cursos de salud. Utilizar estrategias que los sensibilicen y creen un enfoque sobre el uso racional de los medicamentos en disciplinas transversales a lo largo de la graduación.

Palabras clave: Salud pública; Estudiantes; Factores de riesgo; Automedicación.

\section{Introdução}

A automedicação é considerada um elemento de autocuidado. Realizada por meio do uso de medicamentos considerados confiáveis, seguros e acessíveis (OMS, 2005). No entanto, vale salientar que o uso de medicamentos tarja vermelha ou preta é restrito à prescrição médica. Diante da magnitude epidemiológica, a prática de automedicação entre universitários, passa a ser vista como um dos principais problemas de saúde pública no Brasil (Gama \& Secoli, 2017).

A automedicação é praticada independente da classe social e econômica do indivíduo, no entanto alguns estudos demonstram que ela tem sido recorrente em pessoas que possuem maior nível de informação. Esse poder de conhecimento, principalmente entre os universitários, proporciona maior confiança em se automedicarem (Sousa \& Sena, 2017).

Alguns estudos descritos na literatura, apontaram uma alta prevalência da automedicação entre universitários de diferentes cursos. Gama e Secoli observaram em estudos que a predominância dessas taxas se situam entre 38,0 \% e 97,8 \% variando de acordo com cada país de origem dos universitários, o curso e período recordativo da automedicação. Os autores afirmam ainda, que a ocorrência da automedicação na Região Norte do país é pouco conhecida, principalmente devido as dimensões geográficas, a limitação de acesso aos serviços de saúde e também devido a influência da cultura indígena nos hábitos da população, especialmente no que tange ao uso de plantas medicinais (Santos et al. 2018; Gama \& Secoli 2017).

Foi observado que os universitários fazem o uso da automedicação para tratar problemas relacionados a sintomas de cefaleia, gripes, inflamação e dores gerais, destacando principalmente os medicamentos pertencentes as classes dos analgésicos, antipiréticos e anti-inflamatórios (Sousa \& Sena, 2017). De acordo com Tognoli et al. (2019) os estudos que envolvem a automedicação entre os universitários das ciências da saúde, possuem a expectativa de que estes tenham uma atitude condizente com a sua formação profissional e sua prática diária, assim como também estejam preparados para orientar sobre o uso racional de medicamentos e que realizem a prática de maneira correta. Contudo, a prática de automedicação tem sido relacionada aos casos de intoxicações e reações adversas a medicamentos, tornando-se uma importante causa de hospitalização e mortalidade. A reação adversa a medicamentos (RAM) é qualquer resposta prejudicial ou indesejável, não intencional, a um medicamento, que ocorre nas doses usualmente empregadas nos indivíduos (Santos \& Boing, 2018). 
A automedicação entre universitários têm sido amplamente estudada em países da Europa, Ásia e América do Norte. Contudo, no Brasil, ainda há poucos estudos sobre a temática. Visto que a avaliação do consumo de medicamentos sem prescrição médica contribui para a aplicação e desenvolvimento de futuros estudos de intervenções, torna-se extremamente necessário difundir discussões sobre o hábito de se automedicar, enfatizando uma compreensão mais ampla da prática junto aos profissionais da saúde, já que os mesmos podem interferir nesse processo, aliando-se aos pacientes e comunidades (Tarley et al. 2018; Souza et al. 2020). Além disso, como visto em outros estudos, a prática de automedição entre os estudantes têm sido frequente, nesse contexto, esse estudo tem como objetivo analisar os fatores que influenciam na pratica da automediação em universitários e contribuir com reduções de taxas dessa prática e com isso acarretar na diminuição de problemas de saúde pública nos país, logo, também responder a alguns questionamentos: é válido se questionar quais fatores e classes de medicamentos que contribuem para o alto predomínio da automedicação entre os universitários? E entre quais áreas de atuação ela é mais prevalente? Nesse sentido, objetivou-se evidenciar e discutir os dados descritos na literatura acerca da automedicação entre os universitários, bem como analisar os fatores que contribuem para essa prática.

\section{Metodologia}

Nesse estudo foi construído uma revisão integrativa de literatura sobre a prática de automedicação entre estudantes de graduação. De acordo com Souza et al. (2017) a revisão integrativa é construída através da coleta de dados realizada a partir de fontes secundárias, por meio de levantamento bibliográfico e baseado nos relatos científicos descritos na literatura. A revisão integrativa emerge como uma metodologia que compila informações atuais sobre as pesquisas científicas realizadas na área e tem como objetivo buscar as semelhanças e diferenças entre os artigos levantados nos documentos de referência.

As etapas de construção da revisão integrativa foram adotadas conforme metodologia descrita por Lanzoni e Meirelles (2011), seguindo seis etapas, sendo elas: 1) seleção da pergunta de pesquisa; 2) definição dos critérios de inclusão dos estudos e seleção da amostra que será estuda; 3) representação dos estudos selecionados em formato de tabelas, levando em consideração todas as características em comum; 4) análise crítica dos dados encontrados, identificando diferenças e conflitos; 5) interpretação dos resultados e por fim 6) reportar, de forma clara, as evidências encontradas.

A estratégia para o levantamento dos artigos na literatura, foi realizada uma busca nas seguintes base de dados: PubMed®, Google Acadêmico®, Literatura Latino-Americana em Ciências da Saúde (LILACS) e Science Direct. Foram utilizados os descritores em ciências da saúde (DeCS) em português e inglês para realizar a busca dos artigos, sendo eles: "automedicação/Self Medication" e "estudantes/Students". A Tabela 1, apresenta o número total de artigos encontrados nas diferentes bases de dados para cada DeCS que foi utilizado entre os anos de 2017 e 2021. 
Tabela 1 - Número total de artigos encontrados nas bases de dados PubMed®, Google Acadêmico®, LILACS e Science Direct a partir dos DeCS utilizados na busca, artigos de 2017 à 2021.

\begin{tabular}{|c|c|c|c|c|c|c|c|c|}
\hline & \multicolumn{2}{|c|}{ PubMed ${ }^{\circledR}$} & \multicolumn{2}{|c|}{ Google Acadêmico® } & \multicolumn{2}{|c|}{ LILACS } & \multicolumn{2}{|c|}{ Science Direct } \\
\hline $\begin{array}{l}\text { DeCS e ID do } \\
\text { descritor }\end{array}$ & $\begin{array}{l}\text { Total de } \\
\text { artigos }\end{array}$ & $\begin{array}{l}\text { Após busca } \\
\text { refinada }\end{array}$ & $\begin{array}{l}\text { Total de } \\
\text { artigos }\end{array}$ & $\begin{array}{c}\text { Após } \\
\text { busca } \\
\text { refinada }\end{array}$ & $\begin{array}{l}\text { Total de } \\
\text { artigos }\end{array}$ & $\begin{array}{l}\text { Após } \\
\text { busca } \\
\text { refinada }\end{array}$ & $\begin{array}{l}\text { Total de } \\
\text { artigos }\end{array}$ & $\begin{array}{l}\text { Após } \\
\text { busca } \\
\text { refinada }\end{array}$ \\
\hline $\begin{array}{l}\text { Automedicação/Self } \\
\text { Medication } \\
\text { ID: D012651 }\end{array}$ & 2.417 & 463 & 2.580 & 700 & 47 & 17 & 4.901 & 190 \\
\hline $\begin{array}{c}\text { Estudantes/Students } \\
\text { ID: D013334 }\end{array}$ & 112.050 & 7 & 15.000 & 937 & 4.384 & 19 & 339.985 & 198 \\
\hline
\end{tabular}

Fonte: Dados da pesquisa (2021).

Para o cruzamento das palavras, foi aplicado os critérios estabelecidos por Almeida et al. (2021). Foi empregado o termo "AND" (inserção de duas ou mais palavras). Dentre o total de artigos encontrados nas diferentes bases de dados, 55 foram consultados e foram adotados os seguintes critérios para seleção dos artigos: todas as categorias de artigo (original, revisão de literatura, reflexão, atualização, relato de experiência e entre outros); palavras chaves que possuíam relação com o tema do estudo; artigos com resumos e textos completos; publicação nos últimos cinco anos publicados nos idiomas português ou inglês; estudos com metodologia adequada ao objetivo da pesquisa; e por fim os artigos que contivessem em seus títulos ou resumos os seguintes DeSCs que foram utilizados na pesquisa. A opção utilizada na pesquisa foi a expressão "termo exato", associada aos seus respectivos DeSCs.

Os critérios de exclusão dos artigos foram: estudos que não atendessem os critérios de inclusão já supracitados, além dos dados obtidos a partir de pesquisas de teses, dissertações, monografias, cartas ao editor, editoriais, estudos duplicados e com resultados conflitantes. Esses critérios de inclusão e exclusão foram utilizados para elaborar o fluxograma de triagem ilustrado na (Figura 1).

Figura 1. Esquema de seleção dos artigos utilizados na construção do estudo.

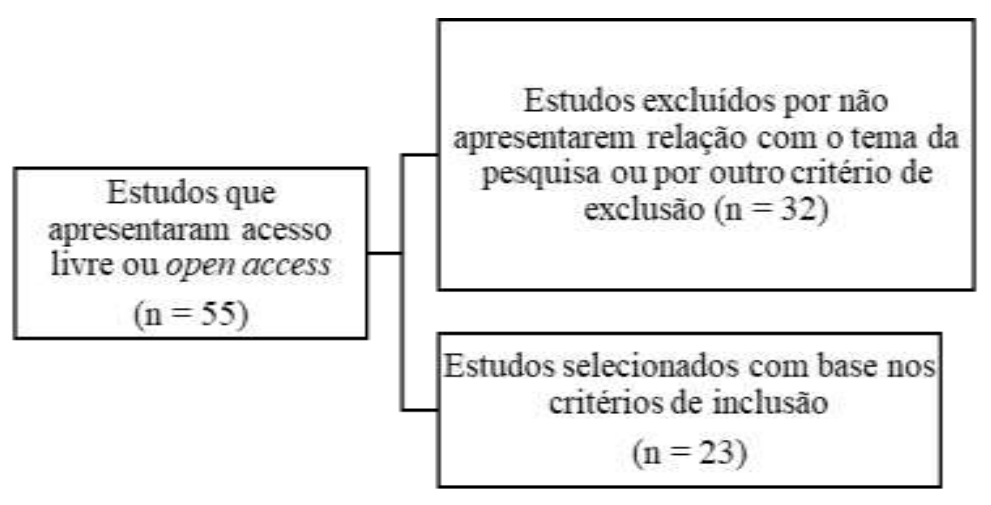

Fonte: Adaptado de Xavier et al. (2021).

Do material obtido, 55 artigos foram lidos minuciosamente e após aplicados os critérios de exclusão, 32 artigos foram excluídos. Seguindo os critérios de inclusão, 23 artigos foram selecionados e fizeram referência ao tema desse estudo, dentre eles, 20 serviram como base para discussão. A partir da leitura dos dados apresentados nos respectivos artigos, os estudos foram agrupados por ordem cronológica, onde foram construídos tabelas e gráficos a fim de comparar os resultados obtidos. 


\section{Resultados e Discussão}

A Tabela 2, foi construída de modo a simplificar as principais informações e proporcionar uma melhor visualização de cada artigo utilizado, dispondo de informações como o nome dos autores, ano de publicação, título, objetivos do estudo e os principais resultados.

De uma forma geral, foi possível observar que em relação aos anos de publicação dos artigos, compreendido entre 2017 a 2021, 30,0 \% foram publicados em 2019, 25,0\% em 2018, 20,0 \% em 2017 e 2020 e 5,0 \% em 2021. Dentre esses estudos $80,0 \%$ (16 artigos) foram publicados em periódicos nacionais e 20,0 \% (4) publicados em periódicos internacionais. Tratando-se da população estudada, houve uma prevalência de 70,0 \% dos universitários pertencentes a área da saúde, principalmente dos cursos de enfermagem, medicina, farmácia e psicologia, e 30,0\% dos estudantes dos cursos de outras áreas.

Tabela 2 - Descrição dos artigos utilizados na construção dessa revisão e principais resultados.

\begin{tabular}{|c|c|c|c|}
\hline $\begin{array}{l}\text { Autores e ano de } \\
\text { publicação }\end{array}$ & Título do artigo & Objetivos do artigo & Principais resultados \\
\hline Sousa \& Sena (2017) & $\begin{array}{l}\text { Automedicação entre universitários } \\
\text { dos cursos de graduação na área da } \\
\text { saúde na FCV-Sete Lagoas: } \\
\text { Influência do conhecimento } \\
\text { acadêmico }\end{array}$ & $\begin{array}{l}\text { Investigar a frequência } \quad \text { da } \\
\text { automedicação e o perfil de } \\
\text { utilização de medicamentos sem } \\
\text { prescrição pelos acadêmicos da } \\
\text { Faculdade Ciências da Vida. }\end{array}$ & $\begin{array}{l}\text { Prevalência de automedicação entre os } \\
\text { universitários de cursos da saúde de } \\
36,1 \% \text {. E } 46,1 \% \text { alegaram não possuir } \\
\text { conhecimento adequado para } \\
\text { automedicação. }\end{array}$ \\
\hline Rathish et al. (2017) & $\begin{array}{l}\text { Pharmacology education and } \\
\text { antibiotic self-medication among } \\
\text { medical students: a cross-sectional } \\
\text { study }\end{array}$ & $\begin{array}{l}\text { Encontrar uma associação entre } \\
\text { educação em farmacologia e } \\
\text { automedicação com antibióticos. }\end{array}$ & $\begin{array}{l}\text { Foi observado que } 28,4 \% \text { faziam o uso } \\
\text { de antibióticos sem prescrição médica, } \\
\text { sendo que } 23,0 \% \text { usam a medicação no } \\
\text { primeiro ano do curso e } 46,0 \% \text { no } \\
\text { quarto ano de curso. }\end{array}$ \\
\hline Gama \& Secoli (2017) & $\begin{array}{l}\text { Automedicação em estudantes de } \\
\text { enfermagem do Estado do } \\
\text { Amazonas-Brasil }\end{array}$ & $\begin{array}{l}\text { Determinar a prevalência e os } \\
\text { fatores associados à automedicação } \\
\text { entre estudantes de enfermagem. }\end{array}$ & $\begin{array}{l}\text { Prevalência de automedicação foi de } \\
76,0 \% \text {, relacionada especialmente com } \\
\text { a percepção de que o problema de } \\
\text { saúde não requeria visita ao médico } \\
(46,6 \%) \text {. }\end{array}$ \\
\hline $\begin{array}{l}\text { Bennett \& Holloway } \\
(2017)\end{array}$ & $\begin{array}{l}\text { Motives for illicit prescription drug } \\
\text { use among university students: A } \\
\text { systematic review and meta- } \\
\text { analysis }\end{array}$ & $\begin{array}{l}\text { Estudar os motivos de estudantes } \\
\text { universitários usarem quatro tipos } \\
\text { diferentes de medicamentos } \\
\text { prescritos } \\
\text { analgésicos, tranquilizantes e } \\
\text { sedativos). }\end{array}$ & $\begin{array}{l}\text { Os motivos para o uso indevido de } \\
\text { medicamentos sem prescrição médica } \\
\text { entre os universitários é a finalidade de } \\
\text { obter aprimoramento pessoal em } \\
\text { relação aos esportes e resultados } \\
\text { acadêmicos, melhorar saúde mental, } \\
\text { reduzir problemas com insônia, } \\
\text { ansiedade e também gerenciar doenças } \\
\text { pré-existentes. }\end{array}$ \\
\hline Luna et al. (2018) & $\begin{array}{l}\text { Consumo de psicofármacos entre } \\
\text { alunos de Medicina do primeiro e } \\
\text { sexto ano de uma Universidade do } \\
\text { Estado de São Paulo }\end{array}$ & $\begin{array}{l}\text { Avaliar a diferença no uso de } \\
\text { drogas psicoativas entre os alunos } \\
\text { do primeiro e sexto ano do curso de } \\
\text { medicina de uma universidade do } \\
\text { interior paulista, identificando os } \\
\text { fatores biopsicossociais associados }\end{array}$ & $\begin{array}{l}\text { Os estudantes do curso de Medicina } \\
\text { dos últimos anos utilizam mais } \\
\text { psicofármacos do que os estudantes no } \\
\text { início do curso, evidenciando a } \\
\text { influência do curso sobrea }\end{array}$ \\
\hline
\end{tabular}


ao uso, compreendendo os fatores medicalização

precipitantes e a ciência dos riscos

de dependência.

Avaliação da prática da Analisar a prevalência da Os resultados obtidos, evidenciaram automedicação em acadêmicos do automedicação em acadêmicos do que 99,5\% dos universitários faziam o

Lima et al. (2018)

curso de Farmácia em uma curso de Farmácia em uma uso da automedicação. instituição privada de ensino instituição privada de Fortaleza, superior em Fortaleza-CE

identificando os principais grupos

terapêuticos envolvidos.

Prática da automedicação entre Caracterizar a prática da Foi observado uma taxa de \begin{tabular}{llll|llll} 
acadêmicos do curso de & automedicação entre os acadêmicos & automedicação & de & $91,2 \%$
\end{tabular}

Santos et al. (2018) enfermagem de uma instituição de ensino superior de Enfermagem de uma Instituição correspondendo a uma frequência de de Ensino Superior. $55,7 \%$.

Estudo comparativo do uso da automedicação entre universitários da área da saúde e universitários de outras áreas não relacionados à saúde na universidade de MaríliaSP

Analisar o uso da automedicação Em ambos os cursos foi identificado entre universitários das áreas da que cerca de 90,0\% dos entrevistados saúde e de outras áreas não fazem o uso da prática e recorrem a relacionados à saúde na automedicação devido a ocorrência de Universidade de Marília -UNIMAR. doenças autolimitadas.

Estudo sobre a automedicação, o uso irracional de medicamentos e o papel do farmacêutico na sua prevenção
Perfil da automedicação em estudantes de ensino superior:

Campos et al. (2019) Impacto na resistência bacteriana
Estabelecer um comparativo entre o uso irracional de antibióticos entre os estudantes da área da saúde e os demais cursos, além de avaliar o profissionais com relação à prática da automedicação.

Self-medication with Antibiotics among University Students in LMIC: A systematic review and meta-analysis

automedicação com antibióticos em estudantes universitários, para explorar as diferenças da

Investigar a prevalência da
automedicação com antibióticos em estudantes de medicina e não médicos, para identificar as razões da automedicação com antibióticos.

Automedicação entre acadêmicos Investigar a automedicação por de Medicina de Fernandópolis -

Tognoli et al. (2019)
São Paulo acadêmicos de curso de graduação em Medicina de instituição privada
Mostrar o papel do farmacêutico na prática da automedicação e como a sua atuação pode influenciar na correta utilização dos medicamentos evitando-se os perigos trazidos pelo o uso irracional. comportamento destes futuros

Os autores identificaram que um dos grandes problemas do uso irracional da medicação é a possibilidade de intoxicação. Cerca de $40,0 \%$ das intoxicações registradas, foram causadas por medicamentos.

Alto índice de automedicação e número significativo de compra de antibióticos sem prescrição médica entre os acadêmicos, além de uma grande tendência na prática da automedicação. A prevalência da automedicação variou entre $11,0 \%$ para os estudantes do Brasil e $90,7 \%$ para os universitários do Congo. O hábito da automedicação aumenta, gradativamente, ao decorrer da graduação.

e analisar possíveis variáveis 
Research, Society and Development, v. 10, n. 8, e47610817594, 2021

(CC BY 4.0) | ISSN 2525-3409 | DOI: http://dx.doi.org/10.33448/rsd-v10i8.17594

\begin{tabular}{|c|c|c|c|}
\hline & & relacionadas. & \\
\hline Colares et al. (2019) & $\begin{array}{l}\text { Prevalence and factors associated } \\
\text { with self-medication in nursing } \\
\text { students }\end{array}$ & $\begin{array}{l}\text { Conhecer a prevalência da } \\
\text { automedicação e os fatores } \\
\text { associados a essa prática entre } \\
\text { estudantes de enfermagem. }\end{array}$ & $\begin{array}{l}\text { Foi observado uma alta prevalência de } \\
\text { automedicação entre os universitários } \\
\text { do curso de Enfermagem, sendo } \\
\text { necessário implantar projeto de } \\
\text { conscientização para que haja um uso } \\
\text { racional dos medicamentos. }\end{array}$ \\
\hline
\end{tabular}

Prevalência da medicalização no Objetiva analisar a prevalência da ensino superior

medicalização em estudantes de ensino superior.
Barbosa et al. (2019)



Carneiro et al. (2019) psicossociais associados em estudantes de medicina no Ceará

(n)

Prevalência da prática de

Souza et al. (2020) automedicação entre estudantes de psicologia: um estudo transversal

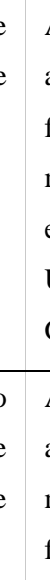

Avaliar a prevalência da prática da automedicação, incluindo o uso de fitoterápicos e/ou plantas medicinais, e os fatores associados, entre os estudantes de psicologia da Universidade Federal de Campina Grande (UFCG).

A prática de automedicação é notada entre os universitários está relacionada com as influencias sociais, culturais e em nível de conhecimento adquiridos no ambiente acadêmico.

Esse estudo objetivou analisar a Estudantes com enxaqueca possuem cefaleia nos estudantes de medicina mais crises antes de provas, tendem a correlacionando-a com períodos de se automedicam mais, fazem o uso provas, fatores psicossociais e horas maior de psicoestimulantes, são mais ansiosos, depressivos, sedentários e também são mais estressados, assim como também dormem menos que aqueles com cefaleia do tipo tensional (CTT)

Foi observado que dentre os estudantes avaliados, $85,4 \%$ fazem o uso de automedicação e $68,7 \%$ pertencem ao gênero feminino.

Avaliar as práticas de $88,0 \%$ dos estudantes se automedicam automedicação em estudantes de e os medicamentos mais utilizados medicina, bem como os fatores, a foram os analgésicos e relaxantes frequência e quais são os musculares. medicamentos mais frequentes.

Automedicação nos estudantes do Esse estudo objetiva caracterizar a Foi observada uma elevada taxa de ensino superior da saúde prática da automedicação nos automedicação (93,4\%). Sendo de estudantes do ensino superior da extrema importância abordar a saúde. problemática ao longo do curso, de forma a reduzir os riscos associados a essa prática.

Prática da automedicação entre estudantes de enfermagem de

\section{instituição de ensino superior}

Bohomol \& Andrade (2020)
Príncipe et al. (2020)

\begin{tabular}{|c|c|}
\hline $\begin{array}{lcc}\text { O objetivo desse estudo } & \text { foi } \\
\text { conhecer a } & \text { prevalência } & \text { dos } \\
\text { medicamentos } & \text { utilizados e } & \text { os } \\
\text { principais } & \text { motivos } & \text { da } \\
\text { automedicação entre os discentes de }\end{array}$ & $\begin{array}{l}\text { Foi observado uma taxa de } \\
\text { automedicação de } 99,2 \% \text { e a classe } \\
\text { medicamentosa prevalente foi a dos } \\
\text { analgésicos. }\end{array}$ \\
\hline
\end{tabular}




\begin{tabular}{|c|c|c|c|}
\hline & & instituição de ensino superior. & \\
\hline Xavier et al. (2021) & $\begin{array}{l}\text { Automedicação e o risco à saúde: } \\
\text { Uma revisão de literatura }\end{array}$ & $\begin{array}{l}\text { Esse estudo objetivou analisar a } \\
\text { prática da automedicação na } \\
\text { sociedade brasileira e entender os } \\
\text { riscos e complicações mais } \\
\text { frequentes dessa prática. }\end{array}$ & $\begin{array}{l}\text { Maior abordagem acerca dos riscos da } \\
\text { automedicação, já que a mesma pode } \\
\text { ocasionar casos extremos até a morte, } \\
\text { visto que todo medicamento tem o } \\
\text { potencial de causar RAM. }\end{array}$ \\
\hline
\end{tabular}

Fonte: Autores.

Com base nos artigos compilados nesse estudo, foi possível observar que dentre as principais causas associadas a prática de automedicação entre os universitários de diferentes áreas de atuação, estão a ocorrência de dores crônicas, acessibilidade dos medicamentos, acreditarem que possuem conhecimento teórico para se automedicarem (principalmente entre os universitários da área da saúde) e até por questões de hábito e entre outros fatores.

Sousa e Sena (2017) a fim de compreender a relação da automedicação dos estudantes aos fatores citados, identificaram que $47,5 \%$ dos universitários adquiriam os medicamentos de acordo com a necessidade e $27,5 \%$ dos entrevistados alegaram se automedicar por possuírem estoque dos medicamentos em casa. No entanto, dentre os universitários que se automedicavam, 2,78\% apresentaram RAM. E quando apresentavam RAM, os indivíduos utilizam os mesmos medicamentos que possuíam em casa ao sentir os mesmos sintomas. Os autores observaram ainda que a prevalência da automedicação entre os universitários foi baixa, representando 36,1\%. Contudo, essa baixa possivelmente está relacionada com o pequeno (n= 36) amostral ou ao fato da população do estudo pertencer aos cursos da área da saúde (Enfermagem, Farmácia, Nutrição e Psicologia), sinalizando que os estudantes apresentaram atitudes coerentes com a formação e profissional.

Como os autores justificaram, a baixa prevalência da automedicação foi considerada baixa em função da pequena população avaliada no estudo, e dentre os fatores que foram destacados como motivos para a prática da automedicação, a acessibilidade do medicamento pode ser visto como um problema recorrente, visto que esse fato está associado com a prática de automedicação em outros estudos que serão abordados ao longo da discussão dessa revisão.

Campos et al. (2019) entrevistaram universitários ( $n=269$ ) dos cursos da área da saúde (grupo A); e de outras áreas (grupo B), buscando estabelecer um comparativo do uso irracional de antibióticos entre eles. Os autores identificaram que 76,5\% (grupo A) e 76,6\% (grupo B) fazem o uso de antibióticos sob orientação médica e 2,4\% utilizam o medicamento sem orientação. Com relação ao hábito de se automedicar, 51,7\% (grupo A) e 58,7\% (grupo B) se automedicam, justificando a prática a influência de amigos, familiares ou por possuírem o medicamento em casa sem prescrição. Após avaliar os dados apresentados nesse estudo, é possível identificar que mesmo que a prevalência de automedicação entre os estudantes dos diferentes grupos sejam próximas, já que outros estudos da literatura apontam uma maior prevalência entre os estudantes da área da saúde, o fato de possuírem medicamentos do tipo antibióticos estocados em casa, adquiridos sem prescrição médica, tem sido recorrente e deve ser tratado com mais atenção.

Luna et al. (2018) avaliaram o uso de medicação controlada entre estudantes universitários do curso de Medicina do primeiro $(n=100)$ e sexto ano $(n=100)$ de uma Universidade de São Paulo. E verificaram que 23,0\% dos estudantes do primeiro e 50,0\% dos alunos do sexto ano, ano faziam uso de psicofármacos, respectivamente. Os estudantes apontaram o curso como principal fator. Contudo, $13,0 \%$ dos estudantes conseguem as receitas para aquisição da medicação de forma ilegal. Como foi possível observar nesse estudo, o aumento da prática da automedicação entre os indivíduos está relacionado com a maior carga de estudos necessário para que os estudantes conduzam o curso e como consequência, faz com que os mesmos passem a recorrer ao uso de medicamentos por conta própria e muitas vezes de forma ilegal. 
Tognoli et al. (2019) ao analisarem a prática da automedicação em estudantes do curso de Medicina $(\mathrm{n}=309)$, observaram resultados superiores em comparação com outros estudos, visto que dentre a população estudada, 96,5\% se automedicava. No entanto, mesmo os estudantes que recorriam a prática, 34,7\% alegaram ter consciência da necessidade de uma prescrição médica, 13,9\% adquiriam informações para se automedicarem com profissionais da saúde. Grande parte dos estudantes avaliados possuíam convênio médico, apresentando concordância com outros dados descritos na literatura. Infelizmente, um dos principais fatores que contribui para essa alta taxa de automedicação entre os estudantes é a dificuldade de obter atendimento médico no sistema de saúde pública.

Tognoli et al. (2019) também perceberam um aumento gradual do percentil de automedicação, associado com o período do curso. Da mesma forma esse comportamento foi observado no trabalho realizado por Rathish et al. (2017) ao entrevistarem universitários do curso de Medicina da Universidade Rajarata do Sri Lanka. Onde foi identificado um aumento na prática da automedicação com base no avanço dos períodos do curso. Nesse estudo, os autores observaram apenas a automedicação aliada ao uso de medicamentos pertencentes a classes dos antibióticos. Esses dados apresentados por Rathish e colaboradores é muito preocupante, visto que o uso de antibióticos pode causar além de reações alérgicas, resistência bacteriana no organismo do indivíduo.

Souza et al. (2020) identificaram uma alta taxa de automedicação entre os estudantes do curso de Psicologia ( $\mathrm{n}=144)$, em que $85,4 \%$ dos indivíduos se automedicavam. Na maioria dos casos, 65,8\% dos estudantes que se automedicavam, buscavam informações com familiares e amigos, e 20,3\% buscavam informações na TV ou pela internet. Entre os problemas relacionados ao uso da medicação, destacaram-se: dor de cabeça, cólica, dores em geral, febre, alergia, dor e inflamação na garganta, gripe e resfriado. Nesse estudo Souza e colaboradores pontuaram que 70,1\% dos estudantes possuem conhecimento acerca dos riscos de se automedicar, no entanto, mesmo assim recorriam a prática. Visto isso, é muito importante que sejam implantadas na grade curricular dos cursos, principalmente cursos da saúde, disciplinas que conscientizem os alunos com relação a prática de se automedicar.

Príncipe et al. (2020) ao analisarem a taxa de automedicação em estudantes de cursos da saúde ( $\mathrm{n}=301)$, verificaram que os sintomas mais frequentes foram as cefaleias $(29,8 \%)$, dismenorreia $(13,5 \%)$ e febre $(9,9 \%)$. Enquanto Carneiro et al. (2019) ao estudarem a prevalência da cefaleia e fatores associados em estudantes de medicina $(n=219)$, constataram que 98,0\% dos indivíduos relataram já ter sentido cefaléia e com relação a prevalência de cefaleia do tipo tensional (CTT) e enxaqueca foi de $61,9 \%$ e $18,1 \%$. Ao comparar esses dados identificados em ambos estudos, é possível identificar que os índices de cefaleia reportado por Carneiro e colaboradores foram superiores aos determinados por Príncipe et al. (2020). Aliado a isso, os indivíduos entrevistados alegaram ter maior intensidade das crises de cefaleia após ingressar no curso.

Lima et al. (2018) encontraram resultados parecidos ao avaliarem a prática de automedicação em acadêmicos do curso de Farmácia ( $n=204)$, identificaram uma maior ocorrência da cefaleia entre os entrevistados $(35,2 \%)$. Esse fato pode estar relacionado às muitas horas de estudo. O que justificaria a maior intensificação das crises entre os estudantes após ingressarem nos cursos de graduação. A Figura 2 ilustra os problemas mais decorrentes associados ao uso da automedicação nos universitários. 
Figura 2 - Percentuais de doenças apresentadas na literatura entre os universitários que fazem o uso da automedicação.

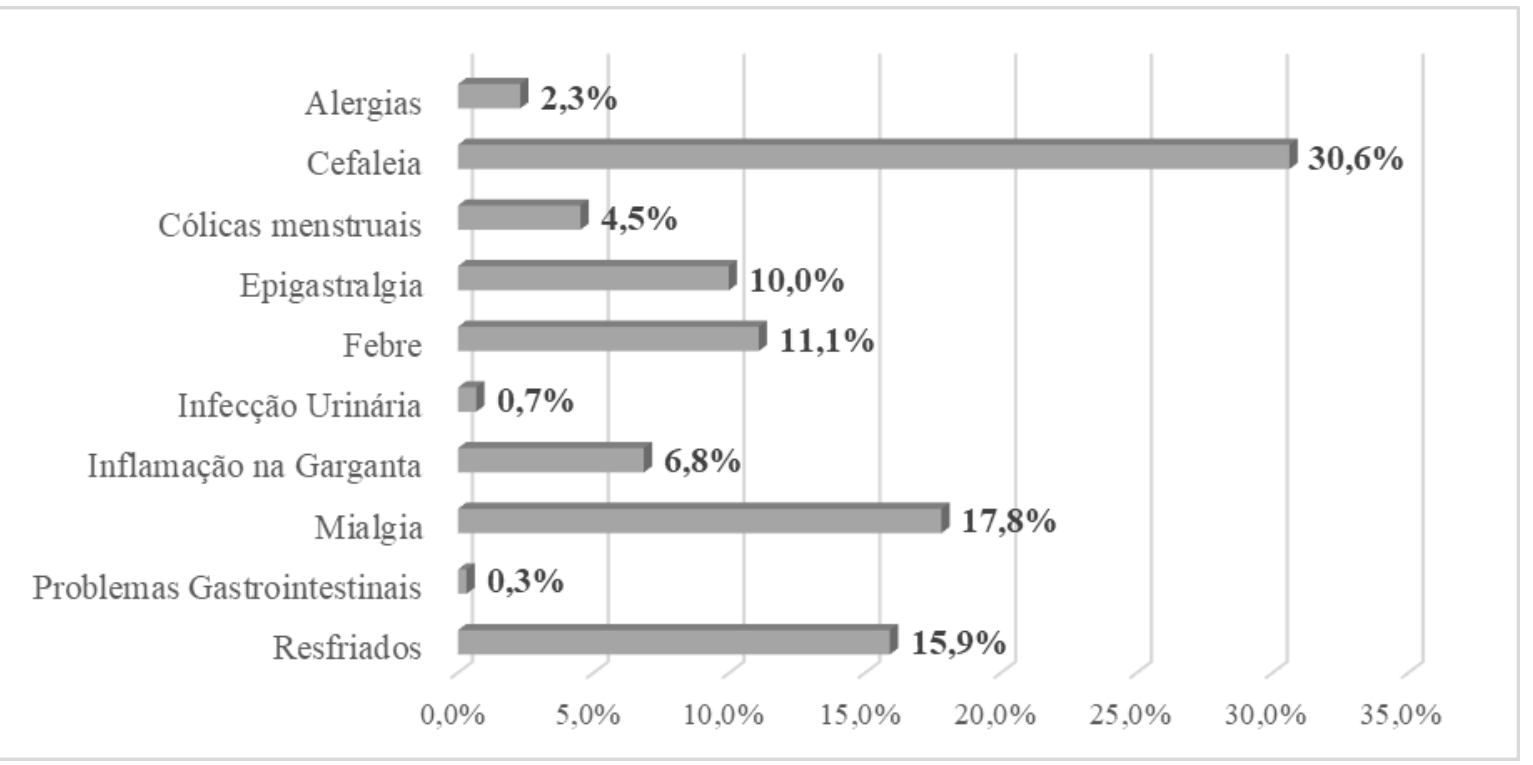

Fonte: Sousa e Sena (2017); Tognoli et al. (2019); Gama e Secoli (2017); Colares et al. (2019); Bennet e Holloway (2017); Lima et al. (2017); Lázaro et al. (2020); Tarley et al. (2018); Souza et al. (2020).

Colares et al. (2019) avaliaram as taxas de automedicação em estudantes de Enfermagem (n=143) e perceberam que as queixas mais comuns como motivos da automedicação relatadas pelos universitários foram dores de cabeça (53,57\%), alergias $(18,57 \%)$ e infecções de garganta (17,14\%). A ocorrência desses sintomas entre os universitários podem ser considerados como alguns dos sintomas físicos de estresse, ocasionado pela intensa rotina de estudos dos indivíduos.

Gama e Secoli (2017) visualizaram que entre os estudantes do curso de enfermagem $(\mathrm{n}=116)$, 50\% deles se automedicavam, e faziam o uso de medicamentos com a finalidade de aliviar dores de cabeça, abdominais e cólicas menstruais. Dentre os medicamentos mais consumidos, foram paracetamol e dipirona $(48,8 \%)$, cefalexina $(6,0 \%)$ e complexo B $(8,3 \%)$. Entre os antibióticos, os mais usados foram cefalexina (55,6\%), amoxicilina $(22,2 \%)$, ampicilina $(11,1 \%)$ e azitromicina $(11,1 \%)$. Como visto nesse estudo, a classes dos medicamentos mais consumidas pelos estudantes foram os analgésicos, provavelmente devido a facilidade na aquisição dos mesmos. Contudo, esse comportamento pode desencadear a incidências de dores crônicas. A causa da dor acaba sendo contornada com o uso dessas drogas e ao utilizar um medicamento especifico de maneira frequente, seu efeito deixa de ser percebido, fazendo com que o indivíduo passe a ingerir doses maiores.

Príncipe et al. (2020) identificaram resultados similares, visto que os medicamentos mais utilizados foram analgésicos (34,6\%), anti-inflamatórios (29,2\%), vitaminas e sais minerais (7,4\%). Barbosa et al. (2019) após avaliar a taxa de automedicação em estudantes de Medicina e Enfermagem verificaram que os analgésicos, corticoide, antiácidos, anticoncepcional, antibiótico, antitérmico e psicofármacos foram os medicamentos mais utilizados na prática de automedicação. A ampla diversidade de medicamentos utilizados na automedicação dos estudantes de cursos da saúde pode ser justificada pela maior confiança em seus conhecimentos adquiridos ao longo da graduação.

Tarley et al. (2018) compararam a taxa de automedicação entre estudantes dos cursos da saúde e cursos de outras áreas $(n=768)$ e perceberam que as doenças relacionadas com a automedicação foram os resfriados, gripes, tosse, febre e dores em geral. Os medicamentos mais utilizados pelos estudantes da área da saúde foram para combater gripes e resfriados, representando 86,7\%, seguidos pelos xaropes para tosse $8,2 \%$, e analgésicos $5,1 \%$, respectivamente. Com relação aos estudantes de outras áreas, os mais consumidos foram medicamentos para resfriado e gripes $89,1 \%$, seguidos por analgésicos $8,3 \%$ e os xaropes para tosse $2,6 \%$. Como visto nesse estudo, o percentual dos medicamentos utilizados pelos estudantes das 
diferentes áreas foram bem similares. Além disso o uso de anti-inflamatórios e descongestionantes é amplamente utilizado na população do estudo, provavelmente por se tratar de medicamentos que podem ser adquiridos facilmente sem prescrição médica.

Os medicamentos aplicados na prática de automedicação são também conhecidos como medicamentos isentos de prescrição (MIPs) e estão disponíveis para comercialização através de uma autorização da Agência Nacional de Vigilância Sanitária (ANVISA) por meio da RDC nº 138/2003. Os MIPs são utilizados para o tratamento de sintomas e são comuns a inúmeros problemas de saúde (Sousa \& Sena, 2017). A Figura 3 ilustra as classes de medicamentos mais usados na automedicação dos universitários de acordo com os dados obtidos na literatura.

Figura 3 - Classes de medicamentos utilizados na automedicação dos universitários.

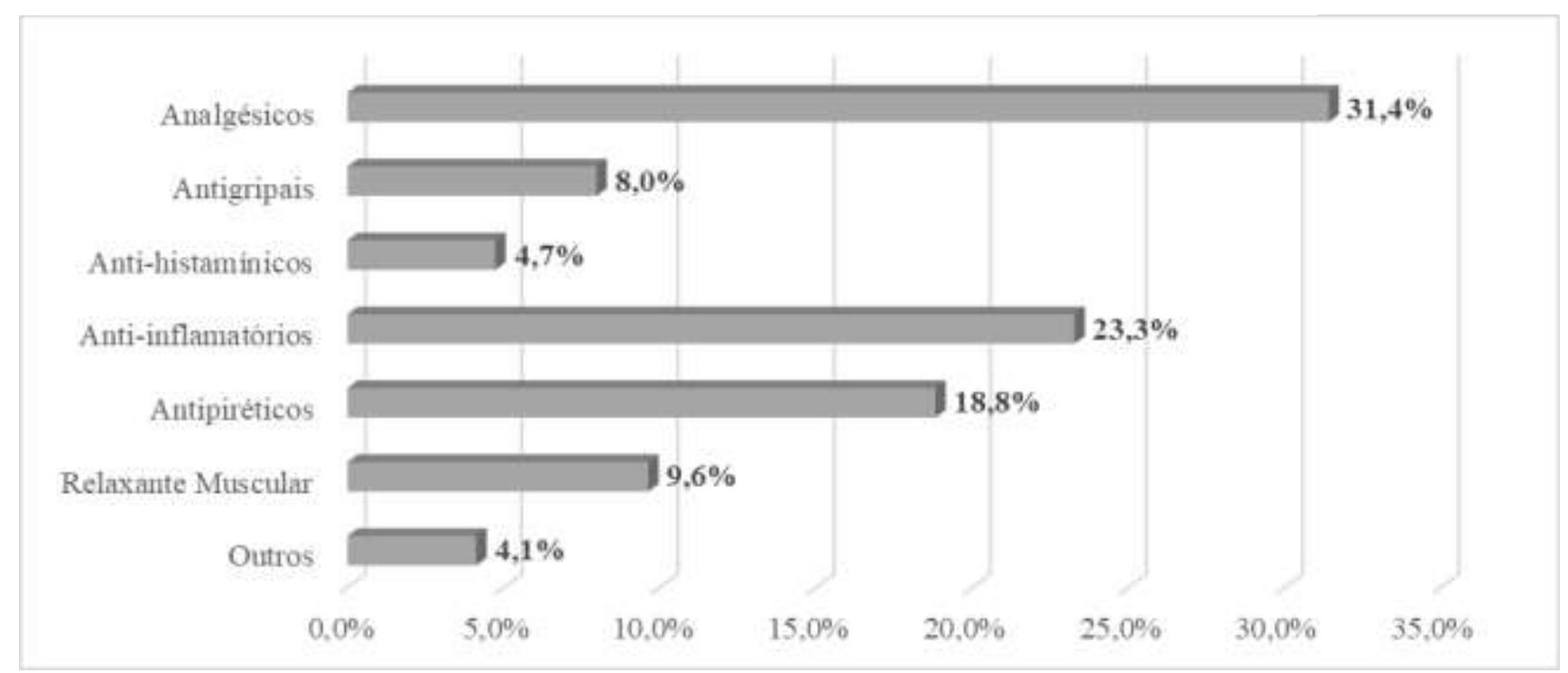

Fonte: Sousa e Sena (2017); Tognoli et al. (2019); Gama e Secoli (2017); Colares et al. (2019); Bennet e Holloway (2017); Lima et al. (2017); Lázaro et al. (2020); Gama e Secoli (2017); Príncipe et al. (2020).

Como visto na Figura 3, mediante os dados reportados na literatura, dentre os medicamentos mais utilizados na automedicação dos universitários, destacaram-se os analgégicos com $(31,4 \%)$ seguido pelos anti-inflamatório não esteroidais (AINES) e os anti-inflamatórios esteroidais (corticoides) com (23,3\%), seguido pelos antipiréticos (15,0\%), os relaxantes musculares $(9,6 \%)$, antigripais $(8,0 \%)$, anti-histamínicos $(4,7 \%)$ e outros medicamentos como (antibióticos, antiespasmódicos, antiparasitários, gastroprotetores), representando cerca de $(4,1 \%)$.

Sousa e Sena (2017) relataram que os medicamentos pertencentes as classes dos antibióticos e antidepressivos são menos utilizados na prática de automedicação dos estudantes, porque precisam de receita médica para serem adquiridos. Em outros estudos reportados na literatura, os estudantes conseguem a prescrição médica para aquisição dessa classe de medicamentos através de amigos e familiares da área da saúde e em alguns casos, interrompem o tratamento antes do período recomendado.

Gama e Secoli (2017) identificaram os antibióticos como o segundo grupo de medicamentos mais utilizados na automedicação dos estudantes do curso de Enfermagem. E esses dados corroboram com outros estudos realizados por países da Ásia e África. Nesses países, a automedicação ocorre por representar uma alternativa viável e de custo mais acessível, visto que muitos estudantes não possuem recursos financeiros para pagarem as consultas médicas ou não tem acesso aos serviços de saúde. Infelizmente esse fato também é recorrente no Brasil, em função dos problemas de desigualdade social existentes no país. Além disso, o uso frequente e indevido dos antibióticos pode levar ao desenvolvimento de bactérias resistentes. 
Rathish et al. (2017) ao determinarem a taxa de automedicação a partir dos antibióticos em estudantes de Medicina, observaram que entre $39,0 \%$ a $95,0 \%$ dos alunos se automedicavam com esse tipo de medicamento, essa prática aumentou progressivamente com o avanço dos períodos cursados. A porcentagem foi significativamente $(\mathrm{p}<0,05)$ maior entre os estudantes da disciplina de Educação em Farmacologia Formal (47,0\%) em comparação aos indivíduos que não cursaram a disciplina $(28,0 \%)$. Um fato que merece destaque nesse estudo, é que os universitários apresentaram uma taxa de automedicação maior ao longo do curso, de acordo com as disciplinas cursadas o que aumenta a carga de conhecimento dos universitários acerca de qual medicamento seria recomendado para cada tipo de doença.

Ferreira e Junior (2018) destacaram a utilização desmedida dos antibióticos, desde os adquiridos através de prescrição médica até os que foram prescritos de maneira indevida, como um dos fatores mais negativos da automedicação entre os estudantes. O uso irracional dos antibióticos ocasiona a disseminação dos genes de resistência. Dentre os medicamentos mais utilizados, os autores estimam que os analgésicos são os mais aplicados na prática. Além desses efeitos negativos reportados por Ferreira e colaboradores, o uso indevido de qualquer medicamento também pode ocasionar outros problemas, como é o caso da intoxicação medicamentosa. Um exemplo prático seria o uso da ivermectina, que é um fármaco utilizado no tratamento de infestações por parasitas, no entanto se utilizada de maneira errada pode causar danos no fígado.

Santos et al. (2018), identificaram os analgésicos e anti-inflamatórios como prevalentes na automedicação dos universitários, onde o paracetamol 75,9\%; dipirona 67,6\%; nimesulida 66,2\%; diclofenaco 55,7\%; vitamina C 55,3\% e 53\% utiliza amoxicilina, foram os mais utilizados na prática.

Lázaro et al. (2020) estudaram a automedicação entre os estudantes do curso de Medicina ( $n=200)$ e encontraram resultados similares aos reportados por Santos e colaboradores, visto que observaram uma maior prevalência dos analgésicos e relaxantes musculares. Bennett e Holloway (2017) investigaram o uso de medicamentos de forma irregular entre os universitários e identificaram a prevalência dos analgésicos, tranquilizantes, sedativos e estimulantes. E foi demonstrado em alguns casos, que os indivíduos fazem o uso dos medicamentos estimulantes a fim de lidar melhor com o estresse acadêmico.

Como citado em alguns estudos ao longo dessa revisão os sintomas físicos de estresse no meio acadêmico contribuem fortemente para a prática de automedicação entre os universitários. Muitas vezes, a ansiedade causada pelas provas e avaliações, problemas com autoestima, problemas econômicos e entre outros fatores, são considerados gatilhos para que esse problema ocorra entre os alunos.

\section{Conclusão}

É possível observar que a prática da automedicação entre os universitários de diferentes áreas têm sido recorrente, principalmente entre os estudantes dos cursos da saúde, em função do maior conhecimento que eles possuem. Investigar e discutir os fatores que contribuem para essa prática é de extrema importância. Já que o consumo de medicamentos de forma irregular e sem prescrição médica pode causar problemas de intoxicações medicamentosas e tem sido considerada como um problema de Saúde Pública.

Dentre as classes de medicamentos mais prevalentes, destacam-se os analgésicos, anti-inflamatórios e os antipiréticos. E quanto aos principais fatores que contribuíram para a automedicação dos estudantes são a alta demanda nos atendimentos do Sistema de Saúde Pública, acessibilidade de compra dos medicamentos sem que haja prescrição médica, influência e indicação dos medicamentos através de amigos ou familiares que atuam na área da saúde, assim como também a disponibilidade de informações nos meios eletrônicos.

Nesse sentido, a partir desse compilado de informações relatados na literatura, foi identificado a necessidade de implantar métodos educativos, principalmente entre os acadêmicos dos cursos da saúde. Utilizando estratégias que 
conscientizem os estudantes e crie uma abordagem sobre o uso racional de medicamentos em disciplinas transversais ao longo da graduação.

Percebe-se a necessidade de desenvolvimento de muitos outros estudos em municípios, regiões e Estados brasileiros com objetivos semelhantes ao abordado nesse manuscrito, esse fator ajudará a ter melhor compreensão do fenômeno da automedicação em universitários em escala maior, o que tende a contribuir com o sanar de problemas no campo da saúde pública.

\section{Agradecimentos}

À Enfermagem Paraibana. À Unidade Acadêmica de Enfermagem (UAENFE) e ao Centro de Educação e Saúde (CES) da Universidade Federal de Campina Grande (UFCG).

\section{Referências}

Almeida, P. T., Teixeira, Y., dos Santos, N. T. Q., Tavares, M. A., Sobreira, L. D. O. A., de Oliveira, R.S., Araújo, Y. T. E., Figueiredo, A. K. G., Sobreira, C. J. R., Menezes, C. M. B., Cunha, L. A. Q. C., Lima, S. C., Araújo, B. C., Lima, R. L., \& Hartcopff, P. F. P. (2021). Utilização de suplementos alimentares por praticantes de atividade física. Research, Society and Development, 10, 12610212355-12610212355.

Barbosa, L. L., Cruz, I. B., Rocha, F. C., dos Santos, T. A. X., Rios, B. M., \& Piris, A. P. (2019). Prevalência da medicalização no ensino superior. Revista Intercâmbio, 15, 35-44.

Bennett, T., \& Holloway, K. (2017). Motives for illicit prescription drug use among university students: A systematic review and meta-analysis. International Journal Drug Policy, 44, 12-22.

Bohomol, E., \& Andrade, C. M. (2020). Prática da automedicação entre estudantes de enfermagem de instituição de ensino superior. Ciência, Cuidado e Saúde, 19, 1-7.

Campos, L. S., Teixeira, B. C., \& Casalini, C. E. C. (2019). Perfil da automedicação em estudantes de ensino superior: impacto na resistência bacteriana. Revista Saúde Integrada, 12, 67-78.

Carneiro, A. F., Neto, P. G. C., Ferreira, J. F. I. S., Garcia, B. F., Silva, F. D. A. C., \& Leal, P. R. L. (2019). A prevalência de cefaleia e fatores psicossociais associados em estudantes de medicina no Ceará. Revista de Medicina, 98, 168-179.

Colares, K. T. P., Barbosa, F. C. R., Marinho, B. M., \& Silva, R. A. R. (2019). Prevalence and factors associated with self-medication in nursing students. Journal of Nursing, 13, 239756-239764.

De Melo Lanzoni, G. M., \& Meirelles, B. H. S. (2011). Liderança do enfermeiro: uma revisão integrativa da literatura. Revista Latino-Americana de Enfermagem, 19, 1-8.

Ferreira, R. L., \& Júnior, A. T. T. (2018). Estudo sobre a automedicação, o uso irracional de medicamentos e o papel do farmacêutico na sua prevenção: Imagem: Vida e Saúde. Revista Científica da Faculdade de Educação e Meio Ambiente, 9, 570-576.

Gama, A. S. M., \& Secoli, S. R. (2017). Automedicação em estudantes de enfermagem do Estado do Amazonas-Brasil. Revista Gaúcha de Enfermagem, 38, $1-7$.

Lázaro, C. A., Gasparini, M. M., Muniz, M. L., Martins, C. D. M., \& Maia, T. A. A. (2020). Investigação sobre a automedicação dos estudantes do curso de medicina de uma instituição de ensino superior. Research, Society and Development, 9, 1-11.

Lima, D. M., da Silva, J. D. S., Vasconcelos, L. F., Cavalcante, M. G., \& Carvalho, A. M. R. (2018). Avaliação da prática da automedicação em acadêmicos do curso de Farmácia em uma instituição privada de ensino superior em Fortaleza-CE. Revista Expressão Católica Saúde, 2, 17-22.

Luna, I. S., Dominato, A. A. G., Ferrari, F., da Costa, A. L., Pires, A. C., \& da Silva Ximendes, G. (2018). Consumo de psicofármacos entre alunos de medicina do primeiro e sexto ano de uma universidade do estado de São Paulo. Colloquium Vitae, 10, 22-28.

Organização Mundial de Saúde (OMS). Dpt. Of Essential Drugs and other Medicines. The role of Pharmacist in selfcare-medication. ttp://www.who.int/medicines/library/docseng_fro m_a_to_z.shtml.

Príncipe, F., Oliveira, A., Silva, C., Silva, D., Silva, D., \& Silva, T. (2020). Automedicação nos estudantes do ensino superior da saúde. Revista de Investigação \& Inovação em Saúde, 3, 21-28.

Rathish, D., Wijerathne, B., Bandara, S., Piumanthi, S., Senevirathna, C., Jayasymana, C., \& Siribaddana, S. (2017). Pharmacology education and antibiotic self-medication among medical students: a cross-sectional study. BMC Res Notes, 10, 1-5.

Santos, G. A. S., \& Boing, A. C. (2018). Mortalidade e internações hospitalares por intoxicações e reações adversas a medicamentos no Brasil: análise de 2000 a 2014. Cadernos de Saúde Pública, 34, e00100917. 
Research, Society and Development, v. 10, n. 8, e47610817594, 2021

(CC BY 4.0) | ISSN 2525-3409 | DOI: http://dx.doi.org/10.33448/rsd-v10i8.17594

Santos, T. S., Almeida, M. M., Pessoa, E. V. M., Pessoa, N. M., Siqueira, H. D. S., Silva, J. M. N., Junior, R. N. C. M., Rodrigues, A. C. E., Silva, F. L., Silva, A. B. S., Pessoa, G. T., \& Sousa, F. D. C. A. (2018). Prática da automedicação entre acadêmicos do curso de enfermagem de uma instituição de ensino superior. Scientia Plena, 14, 1-9.

Sousa, L. A., \& de Andade Sena, C. F. (2017). Automedicação entre universitários dos cursos de graduação na área da saúde na FCV-Sete Lagoas: influência do conhecimento acadêmico. Revista Brasileira de Ciências da Vida, 5, 1-21.

Sousa, L. M. M., Marques-Vieira, C. M. A., Severino, S. S. P., \& Antunes, A. V. (2017). A metodologia de revisão integrativa da literatura em enfermagem. Revista Investigação em Enfermagem 21, 17-26.

Souza, J. F., de Lima, R. M., Batista, J. R. M., \& Mariz, S. R. (2020). Prevalência da prática de automedicação entre estudantes de psicologia: um estudo transversal. Brazilian Journal of Development, 6, 98105-98116.

Tarley, M. G. G., Henrique, E., Miguel, M. A., Costa, M. H., Gonzaga, H. F. S., Carli, F. V. B. O., \& Zutin, T. L. M. (2018). Estudo comparativo do uso da automedicação entre universitários da área da saúde e universitários de outras áreas não relacionados à saúde na Universidade de Marília-SP. Brazilian Journal of Surgery and Clinical Research, 23, 22-27.

Tognoli, T. A., Tavares, V. O., Ramos, A. P. D., Batigália, F., Godoy, J. M. P., \& Ramos, R. R. (2019). Automedicação entre acadêmicos de medicina de Fernandópolis - São Paulo. Journal of Health \& Biological Sciences, 4, 382-386.

Xavier, M. S., Castro, H. N., de Souza, L. G. D., de Oliveira, Y. S. L., Tafuri, N. F., \& Amâncio, N. D. F. G. (2021). Automedicação e o risco à saúde: uma revisão de literatura. Brazilian Journal of Health Review, 4, 225-240.

Xu, R., Mu, T., Wang, G., Shi, J., Wang, X., \& Ni, X. (2019). Self-medication with antibiotics among university students in LMIC: A systematic review and meta analysis. The Journal of Infection in Developing Countries, 13, 678-689. 